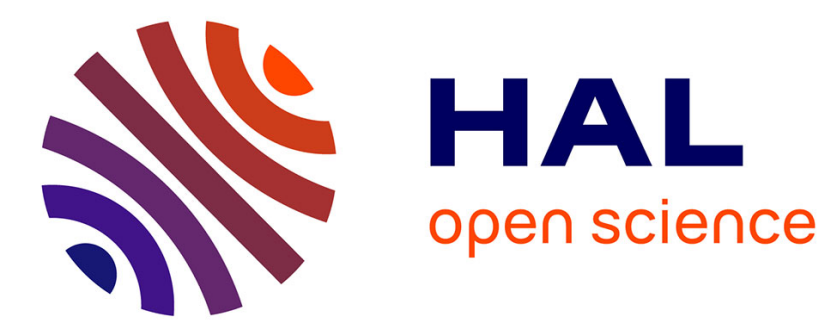

\title{
A Reduction Algorithm for Matrices Depending on a Parameter
}

\author{
Claude-Pierre Jeannerod, Eckhard Pflügel
}

\section{To cite this version:}

Claude-Pierre Jeannerod, Eckhard Pflügel. A Reduction Algorithm for Matrices Depending on a Parameter. International Symposium on Symbolic and Algebraic Computation (ISSAC), Jul 1999, Vancouver, Canada. hal-03419442

\section{HAL Id: hal-03419442 \\ https://hal.inria.fr/hal-03419442}

Submitted on 8 Nov 2021

HAL is a multi-disciplinary open access archive for the deposit and dissemination of scientific research documents, whether they are published or not. The documents may come from teaching and research institutions in France or abroad, or from public or private research centers.
L'archive ouverte pluridisciplinaire HAL, est destinée au dépôt et à la diffusion de documents scientifiques de niveau recherche, publiés ou non, émanant des établissements d'enseignement et de recherche français ou étrangers, des laboratoires publics ou privés. 


\title{
A Reduction Algorithm for Matrices Depending on a Parameter
}

\author{
C.-P. Jeannerod, E. Pflügel \\ LMC-IMAG, 51 Rue des Mathématiques \\ 38041 Grenoble Cedex 9, France \\ $\{$ Claude-Pierre.Jeannerod, Eckhard.Pfluegel\}@imag.fr
}

\begin{abstract}
In this article, we study square matrices perturbed by a parameter $\varepsilon$. An efficient algorithm computing the $\varepsilon$-expansion of the eigenvalues in formal Laurent-Puiseux series is provided, for which the computation of the characteristic polynomial is not required. We show how to reduce the initial matrix so that the Lidskii-Edelman-Ma perturbation theory [16] can be applied. We also explain why this approach may simplify the perturbed eigenvector problem. The implementation of the algorithm in the computer algebra system MAPLE has been used in a quantum mechanics context to diagonalize some perturbed matrices and is available.
\end{abstract}

\section{Introduction}

Let $F=\mathbb{C}[\varepsilon]]\left[\varepsilon^{-1}\right]$ be the field of MFPS (meromorphic formal power series). This paper studies the eigenvalue problem for $A(\varepsilon) \in F^{n \times n}$ from an algorithmic point of view and assumes that the computation of the characteristic polynomial of $A(\varepsilon)$ is not allowed. The MFPS matrix $A(\varepsilon)$ has the form

$$
A(\varepsilon)=\varepsilon^{v} \sum_{j=0}^{\infty} A_{j} \varepsilon^{j} \quad\left(A_{0} \neq 0\right),
$$

with leading term $A_{0}$ of arbitrary Jordan structure, and where $v \in \mathbb{Z}$ is called the valuation of $A(\varepsilon)$. Without loss of generality we may assume that $v=0$, hence

$$
A(\varepsilon)=A_{0}+A_{1} \varepsilon+A_{2} \varepsilon^{2}+\cdots .
$$

The matrix $A(\varepsilon)$ can be seen as a perturbation of $A_{0}$ by order $\varepsilon$. The change of the eigenvalues and eigenvectors of $A_{0}$ (supposed to be nilpotent) under such a small perturbation has been widely studied $[26,23,15,3,13,5]$ and the most recent results are due to the numerical linear algebra community $[17,16]$ : generically, i.e. if we choose $A_{1}, A_{2}, \ldots$ at random, eigenvalues split into rings of radius $O\left(\varepsilon^{\beta}\right), \beta=1 / n$ with $n$ the size of a Jordan block of $A_{0}$. In this case, the eigenvalue problem has been solved by Lidskii [15]. However nongeneric perturbations exist. Among all these nontypical behaviors we distinguish between the case $0 \leq \beta<1$ and the case $\beta \geq 1$. Edelman and Ma [16] have recently shown that in the first category new "generic" behaviors occur, whereas to our knowledge the second one has remained untreated until now. The question is how to use the existing perturbation theory, which we call the Lidskii-Edelman-Ma (LEM) perturbation theory, in order to handle both categories algorithmically and efficiently. Our main result is that every $\beta \geq 1$ case can be reduced to the $0 \leq \beta<1$ case for an appropriate smaller MFPS matrix. To achieve such a reduction process, we define a column reduced by valuation (CRV) form for $A(\varepsilon)$ and generalize a matricial splitting lemma. This leads to an effective algorithm to compute truncated expansions of the eigenvalues of $A(\varepsilon)$, for which the characteristic polynomial of $A(\varepsilon)$ need not be known from the outside. These results also suggest two different approaches for the computation of the $\varepsilon$-expansions of the (generalized) eigenvectors of $A(\varepsilon)$.

Our first motivation was the perturbation study of some matrices $A(\varepsilon)$ used in quantum mechanics, to which Schrödinger perturbation formulas do not apply [12]. Moreover, we wanted to give insight into some particular nongeneric perturbations. In that sense, the matrix [16, example 3]

$$
A(\varepsilon)=J_{5}(0)+\left[\begin{array}{ccccc}
1 & 10 & 3 & -5 & 5 \\
-72 & 19 & -6 & -1 & 9 \\
-12 & 17 & -9 & -4 & 26 \\
0 & 7 & 5 & 6 & -10 \\
0 & 0 & 1 & -6 & -10
\end{array}\right] \varepsilon
$$

whose eigenvalues satisfy $\lambda_{1,2,3}(\varepsilon)=O\left(\varepsilon^{1 / 3}\right.$ ) (ring of radius $\beta=1 / 3)$ and $\lambda_{4,5}(\varepsilon)=\frac{25 \pm \sqrt{7585}}{8} \varepsilon+o(\varepsilon)$, can now be seen as a generic case in practice. To be more precise, eigenvalues $\lambda_{4}(\varepsilon)$ and $\lambda_{5}(\varepsilon)$ can be handled algorithmically. Finally, we wanted to provide an implementation of an algorithm for matrices whose entries are rational functions, which is a subset of the set of MFPS matrices. Hence a MAPLE package, called MP (Matrix Perturbations), and available at http://ww-lmc. imag.fr/ jeannero/.

The eigenvalues of $A(\varepsilon)$ being the roots of its characteristic polynomial $f(\varepsilon, \lambda)=\operatorname{det}(A(\varepsilon)-\lambda I)$, it is well known [24] that each of them can be expressed in some neighborhood of $\varepsilon=0$ as a formal Puiseux series, i.e. each admits a formal expansion in fractional powers of $\varepsilon$. Let $\lambda(\varepsilon)$ be an 
eigenvalue of $A(\varepsilon)$. It can be written

$$
\lambda(\varepsilon)=\sum_{j=0}^{\infty} \lambda_{j} \varepsilon^{j / r}
$$

with $\lambda_{j} \in \mathbb{C}$ and $r \geq 1$ an integer. The leading term $\lambda_{0}$ of $\lambda(\varepsilon)$ is an eigenvalue of the leading matrix $A_{0}$ of $A(\varepsilon)$. The (generalized) eigenvectors $\mathrm{x}_{i}(\varepsilon)$ associated with $\lambda(\varepsilon)$ are solutions of linear systems of the form

$$
(A(\varepsilon)-\lambda(\varepsilon) I) \mathbf{x}_{i}(\varepsilon)=\mathbf{x}_{i-1}(\varepsilon) \quad(i \geq 0)
$$

where $\mathrm{x}_{-1} \equiv 0$. They can be written as vectors of formal Laurent-Puiseux series [3, p.234], i.e.

$$
\mathbf{x}_{i}(\varepsilon)=\varepsilon^{-p_{i}} \sum_{j=0}^{\infty} \mathbf{x}_{i j} \varepsilon^{j / r}
$$

with $\mathrm{x}_{i j} \in \mathbb{C}^{n}$ and $-p_{i} \in \mathbb{Z}$ the valuation or leading exponent of $\mathbf{x}_{i}(\varepsilon)$, with leading coefficient $\mathbf{x}_{i 0} \neq 0$. The $\mathbf{x}_{i}(\varepsilon)$ 's are determined up to multiplication by FPS's. Hence, one may assume that $p_{1}=0$ and $\mathbf{x}_{10} \neq 0$. Then, $\mathbf{x}_{i 0}$ is a (generalized) eigenvector of $A_{0}$ associated with $\lambda_{0}$.

We call approximation up to order $q \in \mathbb{Q}^{+}$of $\lambda(\varepsilon)$ and of the $\mathrm{x}_{i}(\varepsilon)$ 's their expansion truncated at order $o\left(\varepsilon^{q}\right)$ and $o\left(\varepsilon^{-p_{i}+q}\right)$ respectively. These quantities are referred to by $\lambda^{(q)}(\varepsilon)$ and $\mathrm{x}_{i}^{(q)}(\varepsilon)$ and satisfy

$$
\lambda(\varepsilon)=\lambda^{(q)}(\varepsilon)+o\left(\varepsilon^{q}\right) \quad \mathbf{x}_{i}(\varepsilon)=\mathbf{x}_{i}^{(q)}(\varepsilon)+o\left(\varepsilon^{-p_{i}+q}\right) .
$$

In practice, $\mathbb{C}$ is replaced by an appropriate algebraic extension $K$ of $\mathbb{Q}$, and the $\lambda_{j}$ 's and $\mathrm{x}_{i j}$ 's belong to $K$ and $K^{n}$ respectively.

Thus, we actually address the question of determining algorithmically $\lambda^{(q)}(\varepsilon)$ and the $\mathrm{x}_{i}^{(q)}(\varepsilon)$ 's for a given $q \in \mathbb{Q}^{+}$. Our approach is based on recent algorithms for the formal reduction of linear differential systems [2, 19]. Their adaptation to the case of an MFPS matrix $A(\varepsilon)$ provides an effective way to split $A(\varepsilon)$ into submatrices to which the LEM perturbation theory may be applied.

In Section 2 we review two methods to compute approximations (5). The first one is based on the characteristic polynomial of $A(\varepsilon)$. The second one consists in applying the LEM perturbation theory and is the method we use in practice. We also recall some useful notions about regular matrix pencils. Sections 3 and 4 contain our main results: we define a reduced matricial form for MFPS matrices and give an effective way to block-diagonalize such reduced matrices whose leading matrix is nilpotent. A reduction algorithm results from the combination of both techniques (Section 4.3). In Section 5, we will show how approximated eigenvalues and eigenvectors are simultaneoulsy provided when $A(\varepsilon)$ is diagonalizable. The reduction algorithm we propose may also simplify the general case of $A(\varepsilon)$ having a nontrivial Jordan structure.

\section{Preliminaries and previous results}

We denote by first-order term the quantity $\mu \varepsilon^{\beta}$ where $\mu=\lambda_{j}$ is the first nonzero $\lambda_{j}$ in expansion (2). For an eigenvalue $\lambda(\varepsilon)$ with first-order term $\mu \varepsilon^{\beta}$, we say that $\mu$ and $\beta$ are its leading coefficient and leading exponent respectively. We call $O\left(\varepsilon^{q}\right)$ (resp. $o\left(\varepsilon^{q}\right)$ ) eigenvalue an eigenvalue $\lambda(\varepsilon)$ equal to 0 up to $O\left(\varepsilon^{q}\right)$ (resp. o( $\left.\varepsilon^{q}\right)$ ). An MFPS matrix $A(\varepsilon)$ is nilpotent up to order $q$ if it has $o\left(\varepsilon^{q}\right)$ eigenvalues only. We recall in the next two sections why the computation of $\lambda^{(q)}(\varepsilon)$ in (5) essentially consists in determining $\mu \varepsilon^{\beta}$.

\subsection{Computing the characteristic polynomial}

The computation of $f(\varepsilon, \lambda)$ allows to solve the algebraic equation $f(\varepsilon, \lambda)=0$. This may be done by using the Newton algorithm: one computes from the polynomial $f(\varepsilon, \lambda)$ the first-order term $\mu \varepsilon^{\beta}$ of each eigenvalue of $A(\varepsilon)$. The terms of higher order are obtained by repeating the process with $f\left(\varepsilon, \lambda+\mu \varepsilon^{\beta}\right)$. There are efficient algorithms [8] and implementations [22] to solve algebraic equations with polynomial coefficients. They can be used to compute $\lambda^{(q)}(\varepsilon)$. Moreover, all first-order terms can be determined from $f(\varepsilon, \lambda)$. However, the computation of the characteristic polynomial $f(\varepsilon, \lambda)$ of $A(\varepsilon)$ should be avoided, especially if $q$ is small. An efficient way to handle MFPS matrices is then to use lazy evaluation, which takes into account only a finite number of terms of $A(\varepsilon)$. Once the eigenvalues have been approximated, eigenvector computations consist in solving linear systems of size $n$ up to order $q$. (We will come back to this point in Section 5.2).

\subsection{On the LEM perturbation theory}

Another approach consists in determining nondegeneracy conditions on the first terms $A_{0}$ and $A_{1}$ of $A(\varepsilon)$, under which $\mu \varepsilon^{\beta}$ can be directly computed (see e.g. [3] $\S 7,[17]$ ). The process is then repeated with the matrix $A(\varepsilon)-\mu \varepsilon^{\beta} I$. The first complete results in that direction were obtained by Lidskii [15]. They also allow the computation of perturbation series for the eigenvectors of $A(\varepsilon)$ associated with simple eigenvalues.

For our algorithm we will use, when possible, the extension of Lidskii's results to perturbed eigenvalues due to Edelman and Ma [16]. They provide algebraic conditions on certain entries of $A_{1}$, which depend both on the Jordan structure of $A_{0}$ and on the sparsity structure of $A_{1}$. Their nondegeneracy conditions generalize the ones initially given by Lidskii. However, they are given in the case when all the Jordan blocks of $A_{0}$ have the same dimension and when $A_{1}$ has upper $k$-Hessenberg blocks, i.e. $A_{0} \sim \operatorname{diag}\left(J_{1}, J_{2}, \ldots, J_{t}\right)$ where the $J_{i}$ 's are Jordan blocks of size $p(t p=n)$ and

$$
A_{1}=\left[\begin{array}{cccc}
A_{1}^{11} & A_{1}^{12} & \ldots & A_{1}^{1 t} \\
A_{1}^{21} & A_{1}^{22} & \ldots & A_{1}^{2 t} \\
\vdots & \vdots & \ldots & \vdots \\
A_{1}^{t 1} & A_{1}^{t 2} & \cdots & A_{1}^{t t}
\end{array}\right]
$$

where every $A_{1}^{i j}$ is an upper $k$-Hessenberg matrix. ( $A_{0}$ is supposed to be nilpotent without loss of generality.) If the Jordan structure of $A_{0}$ consists of blocks of various dimensions, there is no general formula making use of the sparsity structure of $A_{1}$ and we restrict ourselves to Lidskii's nondegeneracy conditions, as reviewed in [17]. Notice that when some conditions are not satisfied, the number of $\mu \varepsilon^{\beta}$ 's we get is strictly smaller than $n$.

It has been shown that the above structure (6) is invariant under similarities $Q$ such that $Q^{-1} A_{0} Q$ is in Jordan normal form [11]. Thus, in practice, the LEM perturbation theory may be used as follows. 
Input: $A(\varepsilon) \in F^{n \times n}$.

Output: a subset of the set of all leading terms $\mu \varepsilon^{\beta}$.

$A_{0} \leftarrow \operatorname{Jordan}\left(A_{0}, Q\right)$

$A_{1} \leftarrow Q^{-1} A_{1} Q$

Init $k$ depending on the sparsity structure of $A_{1}$;

Compute the algebraic conditions according to

[16, pp.59-61] or [17, p.798];

For every satisfied condition, return the corresponding leadings terms;

As stated in the introduction, we will use this algorithm after having transformed $A(\varepsilon)$. We will see in Section 3 that the effect of such a transformation is easier to see if one rewrites $A(\varepsilon)$ as a regular matrix pencil. Hence a few reminders on the subject (see e.g. [9] for more details).

\subsection{Regular matrix pencils}

Let $N$ and $D$ be $n$ by $n$ square matrices with coefficients in $F$ and $\lambda$ an indeterminate. The matrix $N-\lambda D$ is called a regular matrix pencil if $\operatorname{det}(N-\lambda D) \not \equiv 0$. The roots of the polynomial $P(\lambda)=\operatorname{det}(N-\lambda D)$ are called the $f-$ nite eigenvalues of the regular matrix pencil $N-\lambda D$. If $d=\operatorname{deg}(P)<n$, the pencil has an infinite eigenvalue of multiplicity $n-d$. If $D=I$ (identity matrix), the finite eigenvalues are the eigenvalues of $N$ in the usual sense. In this case and more generally if det $D \neq 0$, one has $d=n$ and the multiplicity of the eigenvalue at infinity is zero. Using multiplication on the left and on the right by invertible constant square matrices leads to the Weierstrass normal form for regular pencils [9]. It has the form $\operatorname{diag}(I-\lambda H, J-\lambda I)$, where $H$ is an $n-d$ by $n-d$ nilpotent matrix in Jordan normal form and $J$ a $d$ by $d$ matrix in Jordan normal form. Notice that the polynomial $P$ is the characteristic polynomial of the matrix $J$. However, in practice one avoids the computation of the Weierstrass normal form, for it may introduce algebraic extensions of the field considered. Instead, we will use a rational block-diagonal form [19]

$$
\left[\begin{array}{ll}
N^{11}-\lambda D^{11} & N^{22}-\lambda D^{22}
\end{array}\right]
$$

such that $\operatorname{det}\left(N^{11}-\lambda D^{11}\right) \equiv 1$ and $\operatorname{det}\left(N^{22}-\lambda D^{22}\right) \equiv P$. This allows to separate the infinite and finite eigenvalues of the regular matrix pencil $N-\lambda D$.

\section{A column reduced (by valuation) form}

We introduce here the matricial form for $A(\varepsilon)$ which is at the root of our reduction algorithm. The computation of such a form provides both an integer inferior bound for the smallest leading exponent, and a polynomial we will use in Section 4 to split this form.

Definition 1 A matrix $A(\varepsilon) \in F^{n \times n}$ is said to be column reduced by valuation (CRV) if it has both a maximal valuation $v$ and a minimal number of nonzero columns of valuation $v$, among all similar matrices.

The valuation of a CRV matrix is smaller than the smallest leading exponent of the eigenvalues. If the valuation is strictly smaller than the smallest leading exponent, the leading matrix $A_{0}$ is nilpotent, and we have no information on the first-order terms of the eigenvalues. However, when $A(\varepsilon)$ is in CRV form, we can often say more. Indeed, if the smallest exponent is an integer value, and if the valuation $v$ is maximal, then both quantities are equal and the nonzero eigenvalues of $A_{0}$ are the leading coefficients of the eigenvalues of $A(\varepsilon)$ of valuation $v$.

Minimizing the number of columns of smallest valuation is inspired by Moser's reduction [18] for linear differential systems in the neighborhood of a singular point. Its main interest is illustrated by Proposition 1. Moser provided an algorithm whose adaptation to the algebraic case, called algebraic-Moser [11], computes an invertible polynomial matrix $T(\varepsilon)$ such that the resulting transformed matrix $\tilde{A}(\varepsilon)=T^{-1}(\varepsilon) A(\varepsilon) T(\varepsilon)$ has a minimum number of columns of valuation, the valuation of $A(\varepsilon)$. When the minimum is zero, the valuation has increased and one may iterate on $\tilde{A}(\varepsilon)$. Otherwise, the maximal valuation has been obtained and $\tilde{A}(\varepsilon)$ is in CRV form. Following Moser [18, p.387], it can be shown that a minimization criteria is that the polynomial

$$
\theta(\lambda)=\left.\varepsilon^{\operatorname{rank}\left(A_{0}\right)} \operatorname{det}\left(\frac{A_{0}}{\varepsilon}+A_{1}-\lambda I\right)\right|_{\varepsilon=0}
$$

does not vanish identically in $\lambda$. Hence we obtain the following algorithm to compute a CRV form of $A(\varepsilon)$ :

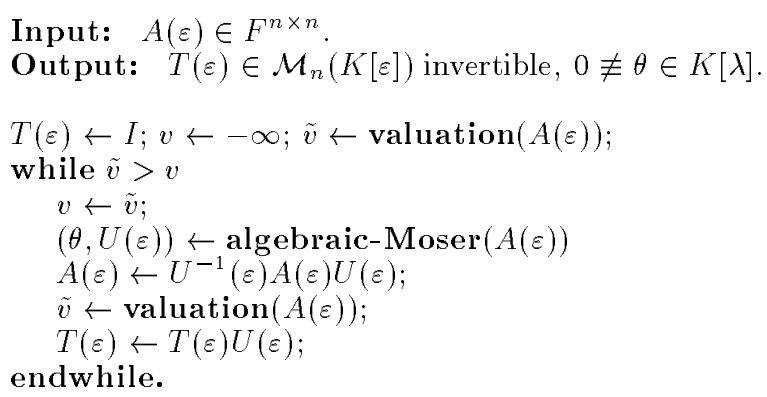

The CRV form may be seen as a special case of the classical CR (column reduced) form for polynomial matrices [27, p.27], where one considers the point at infinity. Furthermore, we work with similarity transformations instead of elementary column transformations.

Proposition 1 Let $A(\varepsilon)$ be in $C R V$ form and $\theta$ be its associated polynomial. Let $\mu$ be a root of $\theta$ with multiplicity $m$. Then $A(\varepsilon)$ has $m$ eigenvalues of the form

$$
\lambda(\varepsilon)=\mu \varepsilon^{v+1}+o\left(\varepsilon^{v+1}\right) .
$$

Proof Let $C_{i}$ denote the $i$-th column of $A(\varepsilon)$ and $\operatorname{val}\left(C_{i}\right)$ its valuation. It will prove convenient to write

$$
A(\varepsilon)=\varepsilon^{v+1} N(\varepsilon) D^{-1}(\varepsilon)
$$

with

$$
D^{-1}(\varepsilon)=\operatorname{diag}\left(\varepsilon^{\alpha_{1}}, \ldots, \varepsilon^{\alpha_{n}}\right)
$$

and where the $\alpha_{i}$ 's are defined by

$$
\alpha_{i}=\min \left(0, \operatorname{val}\left(C_{i}\right)-v-1\right) .
$$


Notice that $N(\varepsilon)$ is an FPS matrix, and

$$
\operatorname{det} D(\varepsilon)=\varepsilon^{\operatorname{rank}\left(A_{0}\right)} .
$$

This allows to express the eigenproblem $\operatorname{det}(A(\varepsilon)-\lambda I)=0$ in terms of the generalized eigenproblem $\operatorname{det}\left(\varepsilon^{v+1} N(\varepsilon)-\right.$ $\lambda D(\varepsilon))=0$. Indeed, the eigenvalues of the regular matrix pencil

$$
N(\varepsilon)-\lambda D(\varepsilon)
$$

are exactly the eigenvalues of $A(\varepsilon)$ mutiplied by $\varepsilon^{v+1}$. The associated polynomial $\theta$ can now be computed from the leading term of $N(\varepsilon)-\lambda D(\varepsilon)$ :

$$
\begin{aligned}
\operatorname{det}\left(N_{0}-\lambda D_{0}\right) & =\left.\operatorname{det} D(\varepsilon) \operatorname{det}\left(\frac{A(\varepsilon)}{\varepsilon^{v+1}}-\lambda I\right)\right|_{\varepsilon=0} \\
& =\left.\varepsilon^{\operatorname{rank}\left(A_{0}\right)} \operatorname{det}\left(\frac{A_{0}}{\varepsilon}+A_{1}-\lambda I\right)\right|_{\varepsilon=0} \\
& =\theta(\lambda),
\end{aligned}
$$

and it is well known [3, Theorem 1 p.351] that the constant terms of the finite eigenvalues of the pencil $N(\varepsilon)-\lambda D(\varepsilon)$ are the finite eigenvalues of its leading pencil $N_{0}-\lambda D_{0}$. $\square$

Hence, we know precisely that there are $n-\operatorname{deg} \theta$ eigenvalues of $A(\varepsilon)$ whose leading exponent lies in $[v, v+1[$, and $\operatorname{deg} \theta O\left(\varepsilon^{v+1}\right)$ eigenvalues. This is illustrated by Example 1. A particular case occurs when $\theta$ is a (nonzero) constant.

\section{Example 1 Consider the companion matrix}

$$
A(\varepsilon)=\left[\begin{array}{ccccc} 
& & & & -2 \varepsilon^{10} \\
1 & & & & 2 \varepsilon^{7} \\
& 1 & & & -3 \varepsilon^{5} \\
& & 1 & & \varepsilon^{3} \\
& & & 1 & -\varepsilon^{2}
\end{array}\right]
$$

whose five eigenvalues satisfy

$$
\begin{array}{r}
\lambda_{1,2}(\varepsilon)= \pm \varepsilon^{3 / 2}+o\left(\varepsilon^{3 / 2}\right), \quad \lambda_{3}(\varepsilon)=\varepsilon^{2}+o\left(\varepsilon^{2}\right), \\
\lambda_{4}(\varepsilon)=2 \varepsilon^{2}+o\left(\varepsilon^{2}\right), \quad \lambda_{5}(\varepsilon)=o\left(\varepsilon^{2}\right) .
\end{array}
$$

One can verify that $\theta \equiv 0$. Reducing $A(\varepsilon)$ yields the transformation matrix

$$
T(\varepsilon)=\left[\begin{array}{ccccc}
\varepsilon^{7} & 0 & -\varepsilon^{7} & 4 \varepsilon^{7} & -6 \varepsilon^{7} \\
0 & \varepsilon^{6} & 0 & -\varepsilon^{5} & 4 \varepsilon^{5} \\
0 & 0 & \varepsilon^{4} & 0 & -\varepsilon^{3} \\
0 & 0 & 0 & \varepsilon^{2} & 0 \\
0 & 0 & 0 & 0 & 1
\end{array}\right]
$$

such that $\tilde{A}(\varepsilon)=T^{-1}(\varepsilon) A(\varepsilon) T(\varepsilon)$ is of valuation 1 . Because of $\lambda_{1,2}$, this value is maximal among all the matrices similar to $A(\varepsilon)$. The associated polynomial is $\theta(\lambda)=$ $-\lambda(\lambda-1)(\lambda-2) \not \equiv 0$. Thus, $\tilde{A}(\varepsilon)$ is in CRV form and the roots of its associated polynomial $\theta$ appear in accordance with Proposition 1 as the leading (possibly zero) coefficients of the eigenvalues $\lambda_{3}, \lambda_{4}$ and $\lambda_{5}$. We will see in Section 4.2 that since $n=5$ and $d=\operatorname{deg} \theta=3>0, \tilde{A}(\varepsilon)$ can be block-diagonalized into two square matrices of respective dimensions $n-d=2$ and $d=3$.

\section{Computing perturbed eigenvalues}

We can now describe a reduction algorithm which computes approximation $\lambda^{(q)}(\varepsilon)$ of $(5)$. At each step, the CRV form is computed and, in order to apply the LEM theory, the resulting matrix is block-diagonalized (or "split") into two blocks. This splitting result is shown in Lemma 1 . We begin with the three following elementary operations which will be used throughout the reduction algorithm:

O1 Similarity transformation with polynomial transformation matrices. The eigenvalues are preserved by this operation.

O2 Substitution $\varepsilon=c \tilde{\varepsilon}^{r}$ for a number $c \in \mathbb{C}$ and $r \in \mathbb{N}^{*}$. The new matrix is $\tilde{A}(\tilde{\varepsilon})=A\left(c \tilde{\varepsilon}^{r}\right)$. Such an operation occurs when an eigenvalue contains a ramification (see Section 4.2). The method we use to compute the value of $c$ is borrowed from Duval [8]. It is motivated by efficiency reasons and leads to minimal algebraic extensions of the field $K$.

O3 Change of eigenvalue $\lambda(\varepsilon)=\mu \varepsilon^{\beta}+\tilde{\lambda}(\varepsilon)$ where $0 \neq \mu \in$ $K$. Using operation $\mathrm{O} 2$ one can suppose that $\beta$ is an integer. The new matrix is given by $\tilde{A}(\varepsilon)=A(\varepsilon)-$ $\mu \varepsilon^{\beta} I$.

Lemma 1 Let $N(\varepsilon)-\lambda D(\varepsilon)$ be a regular FPS matrix pencil with leading term

$$
N_{0}-\lambda D_{0}=\left[\begin{array}{ll}
N_{0}^{11}-\lambda D_{0}^{11} & N_{0}^{22}-\lambda D_{0}^{22}
\end{array}\right] .
$$

If $N_{0}^{11}-\lambda D_{0}^{11}$ and $N_{0}^{22}-\lambda D_{0}^{22}$ are regular matrix pencils with disjoint spectra, then there exist invertible FPS matrices

$$
S(\varepsilon)=I+S_{1} \varepsilon+\cdots, \quad T(\varepsilon)=I+T_{1} \varepsilon+\cdots,
$$

such that

$$
\tilde{N}(\varepsilon)-\lambda \tilde{D}(\varepsilon)=T^{-1}(\varepsilon)(N(\varepsilon)-\lambda D(\varepsilon)) S(\varepsilon)
$$

is block-diagonal with the same structure as $\tilde{N}_{0}-\lambda \tilde{D}_{0}=$ $N_{0}-\lambda D_{0}$.

Proof The proof is essentially the same as the proof originally given by the second author [19, Proposition 2.1] in a differential context. It is constructive and shows how to compute the terms of $T(\varepsilon)$ and $S(\varepsilon)$ successively.

Formally comparing the coefficients of $\varepsilon^{i}(i \geq 1)$ in

$$
(N(\varepsilon)-\lambda D(\varepsilon)) S(\varepsilon)-T(\varepsilon)(\tilde{N}(\varepsilon)-\lambda \tilde{D}(\varepsilon))=0
$$

yields

$$
\begin{aligned}
& N_{0} S_{i}-T_{i} N_{0}-\tilde{N}_{i}=P_{i} \\
& D_{0} S_{i}-T_{i} D_{0}-\tilde{D}_{i}=Q_{i}
\end{aligned}
$$

with $P_{i}, Q_{i}$ depending on terms of $\tilde{N}(\varepsilon), \tilde{D}(\varepsilon), S(\varepsilon)$ and $T(\varepsilon)$ of order lower than $i$. Consider the "ansätze"

$$
S(\varepsilon)=\left[\begin{array}{cc}
I & \mathrm{U}(\varepsilon) \\
\mathbf{V}(\varepsilon) & I
\end{array}\right], \quad T(\varepsilon)=\left[\begin{array}{cc}
I & \mathrm{~W}(\varepsilon) \\
\mathbf{X}(\varepsilon) & I
\end{array}\right]
$$

where $\mathbf{U}(\varepsilon), \mathbf{V}(\varepsilon), \mathbf{W}(\varepsilon)$ and $\mathbf{X}(\varepsilon)$ are FPS matrices of valuation 1 , whose dimensions are given by the block-structure 
of $N_{0}-\lambda D_{0}$. Inserting (11) into (10) leads to the following system of simultaneous matrix equations

$\left(N_{0}^{11}-\lambda D_{0}^{11}\right) \mathbf{W}_{i}-\mathbf{U}_{i}\left(N_{0}^{22}-\lambda D_{0}^{22}\right)=P_{i}^{12}-\lambda Q_{i}^{12}$

$\left(N_{0}^{22}-\lambda D_{0}^{22}\right) \mathbf{X}_{i}-\mathbf{V}_{i}\left(N_{0}^{11}-\lambda D_{0}^{11}\right)=P_{i}^{21}-\lambda Q_{i}^{21}$

with obvious notations. One applies to (12) a result of Chu [7] saying that a simultaneous matrix equation defined by two regular pencils has a unique solution if and only if the spectra of the pencils have an empty intersection. This allows to compute

$$
T_{i}=\left[\begin{array}{ll} 
& \mathbf{U}_{i} \\
\mathbf{V}_{i} &
\end{array}\right], \quad S_{i}=\left[\begin{array}{ll} 
& \mathbf{W}_{i} \\
\mathbf{X}_{i} &
\end{array}\right] .
$$

The result follows by induction.

Lemma 1 generalizes the classic matrix splitting $(N(\varepsilon)=A(\varepsilon), D(\varepsilon)=I,[25,21])$. An algorithm based both on the CRV form and on Lemma 1 can now be designed. It is divided into three sub-cases described in Sections 4.1 and 4.2, where we suppose $A(\varepsilon)$ is in CRV form. The main case distinction is made depending on the structure of the leading matrix $A_{0}$.

\subsection{The matrix $A_{0}$ has several eigenvalues}

This is the classic case. (It has been treated similarly in the differential case $[2,14,20,6]$.) The characteristic polynomial of $A_{0}$ factors in $K$ as

$$
\operatorname{det}\left(A_{0}-\lambda I\right)=\prod_{i=1}^{r_{0}}\left(\lambda-\mu_{i}\right)^{c_{i}} .
$$

By recursively applying Lemma 1 starting with $N(\varepsilon)=A(\varepsilon)$ and $D(\varepsilon)=I, A(\varepsilon)$ is splitted into $r_{0}$ matrices $A^{i i}(\varepsilon)$ of dimension $c_{i}$ with leading matrix admitting $\mu_{i}$ as a unique eigenvalue. If $\mu_{i} \neq 0$, operation $\mathrm{O} 3$ reduces $A^{i i}(\varepsilon)$ to the case of a nilpotent leading matrix. Notice that the knowledge of the splitting field of $\operatorname{det}\left(A_{0}-\lambda I\right)$ is not required in practice, and computations can be done up to conjugation.

\subsection{The matrix $A_{0}$ is nilpotent}

We aim to apply Lemma 1 in a nontrivial way to $A(\varepsilon)$ in CRV form, in order to achieve a further splitting. Write $A(\varepsilon)=\varepsilon^{v+1} N(\varepsilon) D^{-1}(\varepsilon)$ with $D(\varepsilon)$ defined as in $(8)$, and recall that $\theta=\operatorname{det}\left(N_{0}-\lambda D_{0}\right) \not \equiv 0$. Let $d=\operatorname{deg} \theta$.

Case when splitting is possible. If $d>0$, one may assume that $N_{0}-\lambda D_{0}$ is in the rational form (7), i.e.

$$
N_{0}-\lambda D_{0}=\left[\begin{array}{ll}
N_{0}^{11}-\lambda D_{0}^{11} & N_{0}^{22}-\lambda D_{0}^{22}
\end{array}\right] \text {. }
$$

with $\operatorname{det}\left(N_{0}^{11}-\lambda D_{0}^{11}\right) \equiv 1$ and $\operatorname{det}\left(N_{0}^{22}-\lambda D_{0}^{22}\right) \equiv \theta$. The two blocks of $N_{0}-\lambda D_{0}$ are regular pencils with disjoint spectra. Hence, according to Lemma 1, there exist $S(\varepsilon)$, $T(\varepsilon)$ which transform $N(\varepsilon)-\lambda D(\varepsilon)$ into

$$
\left[\begin{array}{ll}
\tilde{N}^{11}(\varepsilon)-\lambda \tilde{D}^{11}(\varepsilon) & \\
& \tilde{N}^{22}(\varepsilon)-\lambda \tilde{D}^{22}(\varepsilon)
\end{array}\right]
$$

with block sizes $n-d$ and $d$. Finally, we obtain

$$
T^{-1}(\varepsilon) A(\varepsilon) T(\varepsilon)=\left[\begin{array}{ll}
\tilde{A}^{11}(\varepsilon) & \\
& \tilde{A}^{22}(\varepsilon)
\end{array}\right]
$$

with

$$
\tilde{A}^{i i}(\varepsilon)=\varepsilon^{v+1} \tilde{N}^{i i}(\varepsilon)\left(\tilde{D}^{i i}(\varepsilon)\right)^{-1}, i \in\{1,2\} .
$$

The $n-d$ by $n-d$ block $\tilde{A}^{11}(\varepsilon)$ is in CRV form, has valuation $v$ and associated polynomial equal to $\operatorname{det}(I-\lambda H) \equiv 1$. Its leading matrix is nilpotent. Consequently, all its eigenvalues have a leading exponent in ]v,v+1[. This case, which appears to be the most difficult, can be handled as described in the next paragraph. The $d$ by $d$ block $\tilde{A}^{22}(\varepsilon)$ has valuation at least $v$ and associated polynomial $\operatorname{det}(J-\lambda I) \equiv \theta$. It may be not in CRV form and its leading matrix may be nilpotent. This matrix has $O\left(\varepsilon^{v+1}\right)$ eigenvalues only, and it can be treated recursively after a possible CRV step.

Example 2 Consider the CRV form provided in the previous example

$$
A(\varepsilon)=\left[\begin{array}{ccccc}
0 & \varepsilon^{2} & -4 \varepsilon^{2} & 6 \varepsilon^{2} & -2 \varepsilon^{3}-6 \varepsilon^{2} \\
\varepsilon & 0 & 0 & 0 & 0 \\
0 & \varepsilon^{2} & 0 & 0 & 0 \\
0 & 0 & \varepsilon^{2} & 0 & 0 \\
0 & 0 & 0 & \varepsilon^{2} & -\varepsilon^{2}
\end{array}\right] .
$$

One has $N_{0}-\lambda D_{0} \sim \operatorname{diag}\left(N_{0}^{11}-\lambda D_{0}^{11}, N_{0}^{22}-\lambda D_{0}^{22}\right)$ where

$$
\begin{gathered}
N_{0}^{11}-\lambda D_{0}^{11}=\left[\begin{array}{cc}
0 & 1 \\
1 & -4-\lambda
\end{array}\right], \\
N_{0}^{22}-\lambda D_{0}^{22}=\left[\begin{array}{ccc}
-1-\lambda & 1 & 0 \\
0 & -\lambda & 1 \\
6 & -6 & 4-\lambda
\end{array}\right] .
\end{gathered}
$$

$N_{0}^{11}-\lambda D_{0}^{11}$ has one double infinite eigenvalue, $N_{0}^{22}-\lambda D_{0}^{22}$ has three finite eigenvalues 0,1 and 2 . By computing (the first terms of) $T(\varepsilon)$ and $S(\varepsilon)$, one splits $A(\varepsilon)$ into 2 by 2 and 3 by 3 blocks satisfying

$$
\begin{array}{r}
\tilde{A}^{11}(\varepsilon)=\left[\begin{array}{cc}
0 & \varepsilon^{2} \\
\varepsilon-10 \varepsilon^{2}-134 \varepsilon^{3} & -4 \varepsilon^{2}-22 \varepsilon^{3}
\end{array}\right]+o\left(\varepsilon^{3}\right), \\
\tilde{A}^{22}(\varepsilon)=\left[\begin{array}{ccc}
-\varepsilon^{2} & \varepsilon^{2} & 0 \\
0 & 0 & \varepsilon^{2} \\
6 \varepsilon^{2}+44 \varepsilon^{3} & -6 \varepsilon^{2}-42 \varepsilon^{3} & 4 \varepsilon^{2}+22 \varepsilon^{3}
\end{array}\right]+o\left(\varepsilon^{3}\right) .
\end{array}
$$

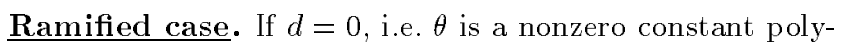
nomial, then all eigenvalues are of the form

$$
\left.\lambda(\varepsilon)=\varepsilon^{v}\left(\mu \varepsilon^{\beta}+o\left(\varepsilon^{\beta}\right)\right), \quad \mu \neq 0, \beta=k / r \in\right] 0,1[.
$$

More precisely, $(k, r) \in \mathbb{N}^{*} \times \mathbb{N}^{*}, r \geq 2$ and $\operatorname{gcd}(k, r)=1$.

The main difficulty is to determine the set $\mathcal{R}$ of all the ramifications $r$ involved. Recall that $n$ denotes the dimension of $A(\varepsilon)$. If $n>3, \mathcal{R}$ is not trivial and the LEM perturbation theory may be used as described in Section 2.2. When a nongeneric case occurs, i.e. we have only a partial knowledge of the whole set $\mathcal{R}$, all the ramifications $r \in\{2, \ldots, n\}$ are systematically considered.

For each ramification $r$ found, operation $\mathrm{O} 2$ allows to apply recursion to $\tilde{A}(\tilde{\varepsilon})$, only looking for eigenvalues of the form

$$
\tilde{\lambda}(\tilde{\varepsilon})=\tilde{\mu} \tilde{\varepsilon}^{k}+o\left(\tilde{\varepsilon}^{k}\right), \quad \tilde{\mu} \neq 0 .
$$




\subsection{Reduction algorithm}

We use splitting and LEM to denote the algorithms associated with Lemma 1 and the LEM perturbation theory respectively. The splitting requires an FPS matrix pencil and a polynomial. In pseudocode we have the following reduction algorithm for computing approximations up to order $q$ of all eigenvalues of $A(\varepsilon)$.

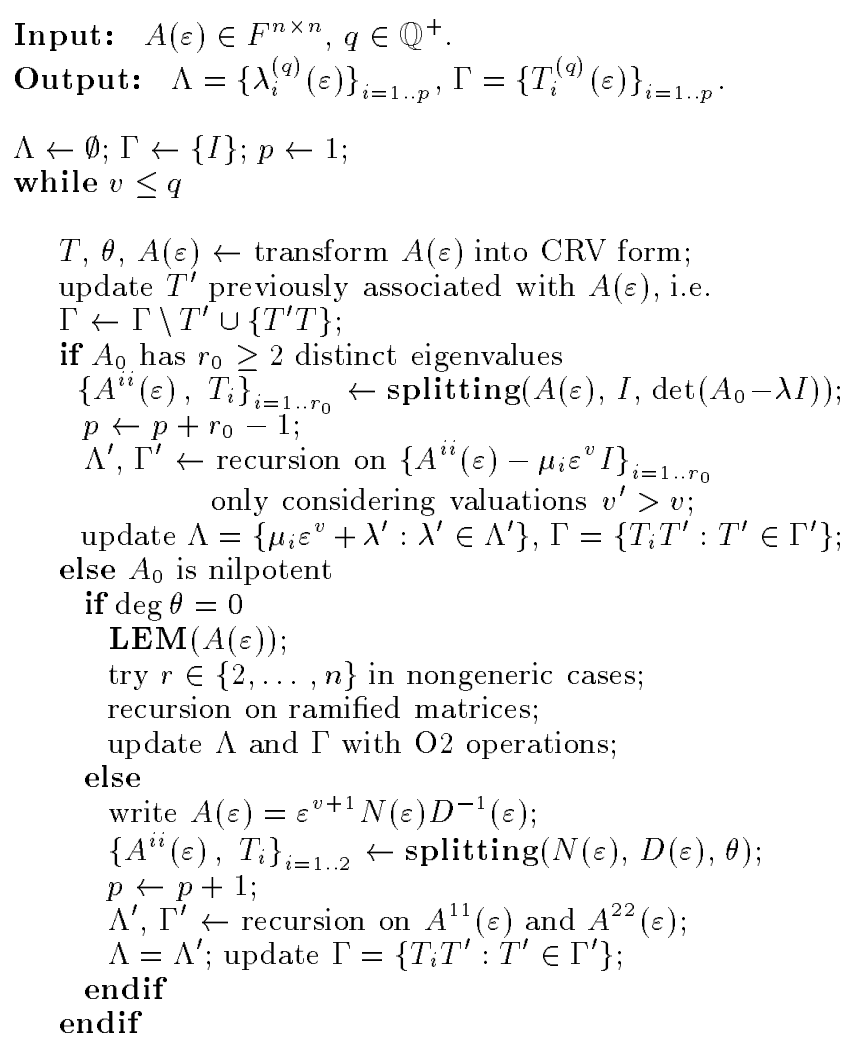

\section{endwhile.}

Notice that the matrices $T$ and $T_{i}$ are all $n$ by $n$ matrices (if necessary, we complete with ones on the diagonal). They depend on $\varepsilon$. Suppose for simplicity that no ramification appears in the eigenvalues. The ouput consists of two sets $\Lambda$ and $\Gamma$, each of size $p<n$. The set $\Lambda$ contains $p$ truncated Laurent-Puiseux series approximating the $n$ eigenvalues $\lambda(\varepsilon)$ up to order $q$. (Two distinct $\lambda(\varepsilon)$ may have the same approximation $\lambda_{i}^{(q)}(\varepsilon)$ and eigenvalues whose coefficients are complex numbers given by roots of unity are counted as a single eigenvalue.) The set $\Gamma$ contains invertible FPS matrices $T_{i}^{(q)}(\varepsilon)$ associated with the $\lambda_{i}^{(q)}(\varepsilon)$ 's and such that

$\left(T_{i}^{(q)}(\varepsilon)\right)^{-1}\left(A(\varepsilon)-\lambda_{i}^{(q)}(\varepsilon) I\right) T_{i}^{(q)}(\varepsilon)=\left[\begin{array}{cc}* & 0 \\ 0 & B_{i}^{(q)}(\varepsilon)\end{array}\right]$

with $B_{i}^{(q)}(\varepsilon)$ of valuation $q$, nilpotent up to order $q$. The size of $B_{i}^{(q)}(\varepsilon)$ equals the multiplicity of $\lambda_{i}^{(q)}(\varepsilon)$.

The following two remarks can be made concerning the efficiency of the algorithm:

- Block-diagonalization provided by Lemma 1 obviously reduces to problems of smaller dimensions. As a consequence, the size of the algebraic extensions effectively used is minimal, and we avoid the introduction of useless ramifications as much as possible.

- Lemma 1 can be specialized to compute upper blocktriangular pencils instead of block-diagonal pencils. The advantage is simpler unimodular transformation matrices, typically

$$
S(\varepsilon)=\left[\begin{array}{cc}
I & \mathrm{U}(\varepsilon) \\
& I
\end{array}\right], \quad T(\varepsilon)=\left[\begin{array}{cc}
I & \mathbf{W}(\varepsilon) \\
I
\end{array}\right] .
$$

Readers familiar with the Newton diagram [3, 17] may also see the reduction algorithm as a way to split the Newton diagram (vertical dashed lines on the diagrams below). It separates integer slopes $v$ from groups of rational slopes lying between two consecutive integer slopes $v$ and $v+1$. The three cases described in Sections 4.1 and 4.2 are respectively:
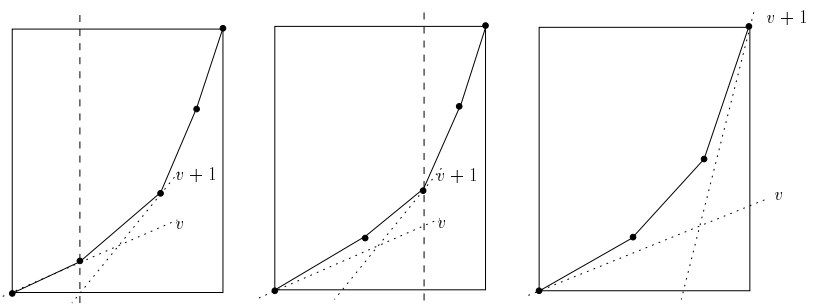

Example 1 corresponds to the second Newton diagram.

The matrix $A(\varepsilon)$ in (1) is seen in [16] as a pathological exception and the LEM theory does not apply to it since one nondegeneracy condition is not satisfied. By applying the reduction algorithm to this problem, we now get the complete solution. Indeed, it block-diagonalizes $A(\varepsilon)$ into two matrices of respective dimensions 2 and 3, whose respective Newton diagrams result from a splitting of the Newton diagram of $A(\varepsilon)$ :

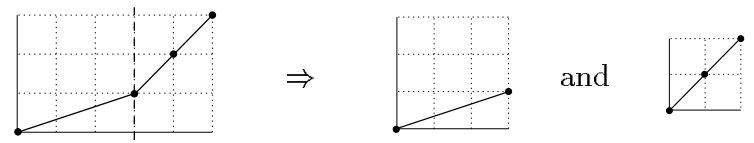

Here, the final valuation is $v=0$ and

$$
\theta(\lambda)=-\left(\lambda-\frac{25+\sqrt{7585}}{8}\right)\left(\lambda-\frac{25-\sqrt{7585}}{8}\right) .
$$

Necessarily, $\mathcal{R}=\{3\}$. Hence the ring of radius $1 / 3$. (The first resulting Newton diagram consists of a single slope 1/3.) The roots of $\theta$ provide the other two eigenvalues, of valuation $v+1=1: \lambda_{4,5}(\varepsilon)=\frac{2.5 \pm \sqrt{7585}}{8} \varepsilon+o(\varepsilon)$. ( $\theta$ is thus the Newton polynomial associated with the slope 1 of the second resulting Newton diagram.)

\subsection{Implementation}

The reduction algorithm has been implemented in the MP package and can be run by calling function eigenvals. This function returns all the $\lambda^{(q)}(\varepsilon)$ 's, together with their multiplicity and their associated block. Further, lazy evaluation for MFPS matrices is the one of ISOLDE package [19, §III]. This technique allows to handle MFPS matrices and to compute a finite number of terms of $S(\varepsilon)$ and $T(\varepsilon)$, which are necessary for reduction up to order $\mathrm{q}$. 
The algorithm has been tested on both polynomial and rational functions matrices $A(\varepsilon)$, whose dimension $n$ ranges from 10 to 100 . Some test-examples are available at http://www-lmc.imag.fr/ jeannero/. In general, our approach proves to be clearly faster than computing the whole characteristic polynomial with Berkowitz algorithm [1]. This is especially true when the approximation order $q$ is small - namely, $q=2$.

\section{Application: eigenvector approximation}

We now consider the possibility of getting some eigenvector approximations by using the reduction algorithm given above. By means of a ramification, we may assume in this section that $r=1$. Let $\lambda^{(q)}(\varepsilon)$ be an approximation of $\lambda(\varepsilon)$, of multiplicity $m$. Recall that, in practice, the reduction algorithm leads to

$$
\left(T^{(q)}(\varepsilon)\right)^{-1}\left(A(\varepsilon)-\lambda^{(q)}(\varepsilon) I\right) T^{(q)}(\varepsilon)=\left[\begin{array}{cc}
* & 0 \\
* & B^{(q)}(\varepsilon)
\end{array}\right]
$$

with $B^{(q)}(\varepsilon)$ of valuation $q$, nilpotent up to order $q$, and of size $m$. By using (3) and setting $\mathrm{x}_{-1}^{(q)}(\varepsilon) \equiv 0$, the approximated eigenvectors defined in $(5)$ can be characterized by

$$
\left(A(\varepsilon)-\lambda^{(q)}(\varepsilon) I\right) \mathbf{x}_{i}^{(q)}(\varepsilon)=\mathbf{x}_{i-1}^{(q)}(\varepsilon)+o\left(\varepsilon^{q}\right) .
$$

\subsection{Semi-simple eigenvalue case}

Assume first that the Jordan canonical form of $A(\varepsilon)$ is diagonal. Then, (14) reduces to

$$
\left(A(\varepsilon)-\lambda^{(q)}(\varepsilon) I\right) \mathrm{x}_{1}^{(q)}(\varepsilon)=o\left(\varepsilon^{q}\right) .
$$

In this case, it can be shown [12] that $B^{(q)}(\varepsilon)$ is 0 up to $o\left(\varepsilon^{q}\right)$. $\left(B^{(q)}(\varepsilon)\right.$ is in Jordan canonical form up to order q.) Consequently, the last $m$ columns of the matrix $T^{(q)}(\varepsilon)$ we compute provide approximations of eigenvectors $\mathrm{x}_{1}(\varepsilon)$. Hence the second function eigenvects added into MP, which collects such eigenvector approximations. Notice that if the valuation $v$ of one of these columns differs from zero, the approximation holds up to order $q-v$.

As a particular case, suppose that $A(\varepsilon)$ is hermitian $\forall \varepsilon \in \mathbb{R}$. Notice that in this situation the eigenelements of $A(\varepsilon)$ contain no ramification (see e.g. [3, p.21], [13, p.120]). Thus, only the CRV form and the classic splitting are needed (Sections 3 and 4.1). In the reduction algorithm, we do not take the most difficult step (Section 4.2), and approximations of both the eigenvalues and the eigenvectors are provided simultaneously. This was used in a semi-conductor context to diagonalize up to order 2 various Kane matrices resulting from the discretization of a Schrödinger operator [12]. A session example is displayed below.

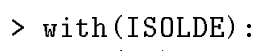

$$
\text { [eigenvals, eigenvects] }
$$

\footnotetext{
$>$ read kane: \# A:= a modified 8 by 8 Kane matrix \# depending on parameter epsilon
}

$>$ eigenvals (A,epsilon, 0,2); \# epsilon $0, q=2$

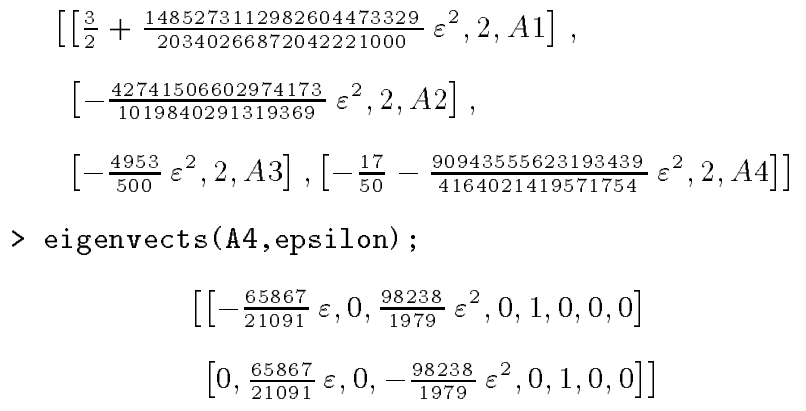

where A4 stands for $B^{(2)}(\varepsilon)(q=2, m=2)$. Since the valuation of these two vectors equals 0 , the algorithm has computed approximations up to order 2 of the eigenvectors associated with the two eigenvalues of $A(\varepsilon)$ whose approximation up to order 2 is $-\frac{17}{50}-\frac{90943555623193439}{4164021419571754} \varepsilon^{2}$. The six other eigenvectors of $A(\varepsilon)^{50}$ can be approximated the same way.

\subsection{General case: a partial result}

Here $B^{(q)}(\varepsilon)$ is no longer in Jordan canonical form and approximated (generalized) eigenvectors can not be retrieved directly from the columns of $T^{(q)}(\varepsilon)$.

Suppose that $m=n$. We want to compute a set of vectors $\left\{\mathrm{x}_{i}^{(q)}(\varepsilon)\right\}$ which satisfy (14). When setting

$$
\mathbf{x}_{i}^{(q)}(\varepsilon)=T^{(q)}(\varepsilon) \mathbf{y}_{i}^{(q)}(\varepsilon) \quad \alpha=q-\text { valuation }\left(T^{(q)}(\varepsilon)\right),
$$

it appears that it is sufficient to solve the problem

$$
B^{(q)}(\varepsilon) \mathbf{y}_{i}^{(q)}(\varepsilon)=\tilde{\mathbf{x}}_{i-1}^{(q)}(\varepsilon)+o\left(\varepsilon^{\alpha}\right)
$$

with $\tilde{\mathbf{x}}_{i-1}^{(q)}(\varepsilon)=T^{(q)}(\varepsilon)^{-1} \mathbf{x}_{i-1}^{(q)}(\varepsilon)$. If $i=1$, this reduces to

$$
B^{(q)}(\varepsilon) \mathbf{y}_{1}^{(q)}(\varepsilon)=o\left(\varepsilon^{\alpha}\right) .
$$

Hence a linear system to be solved up to a given order. If $i \geq 2$, one has to solve a problem of size $n \times(n+1)$ similar to $(15)$ by writing

$$
\left[B^{(q)}(\varepsilon), \tilde{\mathbf{x}}_{i-1}^{(q)}(\varepsilon)\right] \cdot\left[\begin{array}{c}
\mathbf{y}_{i}^{(q)}(\varepsilon) \\
-1
\end{array}\right]=o\left(\varepsilon^{\alpha}\right)
$$

In the general case $m \leq n$, we consider only the last $m$ columns (resp. rows) of $T^{(q)}(\varepsilon)$ (resp. $T^{(q)}(\varepsilon)^{-1}$ ), and we complete the computed eigenvectors with $n-m 0$ 's.

To solve problem (15), one may either use modular fraction-free Gaussian elimination [10], or see (15) as a vector Hermite Padé approximant problem and efficiently compute a $\sigma$-basis for it [4]. However, among the $n$ vectors of the $\sigma$-basis, one should be able to decide which vectors are to be disregarded while iterating the process.

The advantages of this reduction algorithm-based approach in comparison to a direct computation on the matrix $A(\varepsilon)$ are the following: 1) Computations are done on independent systems of smaller dimension. 2) As a result, this reduces the necessary calculations with algebraic numbers and ramifications. 


\section{Conclusions and future directions}

We have shown that using the CRV form of an MFPS matrix $A(\varepsilon)$ leads to a nonclassic splitting of $A(\varepsilon)$. Then, the LEM perturbation theory can be applied to the resulting submatrices. Combining the three CRV, splitting and LEM algorithms provides an efficient way to compute perturbed eigenvalue approximations, using a finite number of terms of $A(\varepsilon)$. Some of the tools used in the reduction algorithm and its implementation are borrowed from both numerical linear algebra and linear differential systems. As an application, we have shown how to approximate the perturbed eigenvectors. When $A(\varepsilon)$ is diagonalizable, both eigenvalues and eigenvectors are provided simultaneously, whereas in the general case, the eigenvectors are obtained after the eigenvalues have been computed.

An ideal algorithmic treatment would compute both eigenvector and eigenvalue $\varepsilon$-expansions at the same time. This has been initiated by Lidskii, Vishik and Lyusternik $[23,15]$ : in [23], a recurrence process is given which provides, for simple eigenvalues only, the set $\left\{\mathrm{x}_{1}^{(q)}(\varepsilon)\right\}$ under some sufficient conditions on $A(\varepsilon)$. However, progressively approximating not only eigenvectors but a Jordan normal form is more difficult since, while running the reduction algorithm, some eigenvector changes may appear between two splitting steps. It would be of interest to provide a generalized reduction algorithm that deals with such discontinuities.

\section{References}

[1] AbDel.Jaoued, J. The Berkowitz algorithm, Maple and computing the characteristic polynomial in an arbitrary commutative ring. Maple Tech \& (3) (1997), 2132.

[2] Barkatou, M. An algorithm to compute the exponential part of a formal fundamental matrix solution of a linear differential system. Journal of App. Alg. in Eng. Comm. and Comp. 8, 1 (1997), 1-23.

[3] BAumgärtel, H. Analytic perturbation theory for matrices and operators. Birkhäuser Verlag, Basel, 1985.

[4] Beckermann, B., and Labahn, G. A uniform approach for the fast computation of Matrix-type Padéapproximants. SIAM J.Matrix Analysis and Applications, 1 (1994), 804-823.

[5] Burke, J., AND Overton, M. Stable perturbations of nonsymmetric matrices. Linear Algebra Appl. 171 (1992), 249-273.

[6] Chen, G. An algorithm for computing the formal solutions of differential systems in the neighbourghood of an irregular singular point. In Proceedings of ISSAC '90 (Tokyo, Japan, 1990), M. N. Shunro Watanabe, Ed., ACM Press, pp. 231-235.

[7] CHU, K. The solution of the matrix equations $A X B-$ $C X D=E$ and $(Y A-D Z, Y C-B Z)=(E, F)$. Linear Algebra Appl. 93 (1987), 93-105.

[8] Duval, D. Diverses questions relatives au calcul formel avec nombres algébriques. Thèse d'Etat, Faculté des Sciences de Grenoble, 1987.
[9] Gantmacher, F. The Theory of Matrices. Volumes 1 and 2, Chelsea, New York, 1959.

[10] Geddes, K. O., Czapor, S., and Labahn, G. Algorithms for Computer Algebra. Kluwer Academic Press, 1992.

[11] Jeannerod, C. Algorithmic reduction of matrices depending on a parameter. Technical Report, LMCIMAG, Grenoble, 1999.

[12] Jeannerod, C., Maillard, N., and Pflügel, E. Using computer algebra to diagonalize some Kane matrices. submitted to J. Phys. A: Math. Gen.

[13] Kato, T. Perturbation theory for linear operators. Springer, Berlin, 1980.

[14] Levelt, A. Jordan decomposition for a class of singular differential operators. Arkiv för matematik 13, 1 (1975).

[15] Linskit, V. Perturbation theory of non-conjugate operators. U.S.S.R. Comput. Math. and Math. Phys. 1 (1965), 73-85.

[16] Ma, Y., AND Edelman, A. Nongeneric perturbations of Jordan blocks. Linear Algebra Appl. 273 (1998), 4563.

[17] Moro, J., Burke, J., and Overton, M. On the Lidskii-Vishik-Lyusternik perturbation theory for eigenvalues of matrices with arbitrary Jordan structure. SIAM J. Matrix Anal. Appl. 18 (1997), 793-817.

[18] Moser, J. The order of a singularity in Fuchs' theory. Math. Z. (1960), 379-398.

[19] PfLügel, E. Résolution symbolique des systèmes différentiels linéaires. Ph.D. Thesis, Université Joseph Fourier, oct. 1998.

[20] Sommeling, R. Characteristic classes for irregular singularities. In Proceedings ISSAC '94 (Oxford, England, 1994), M. Giesbrecht, Ed., ACM Press, pp. 163-167.

[21] Turritin, H. Convergent solutions of ordinary linear homogeneous differential equations in the neighborhood of an irregular singular point. Acta Math. 93 (1955), 27-66.

[22] van HoeiJ, M. The intbasis package. Maple share library.

[23] Vishik, M., And Lyusternik, L. The solution of some perturbation problems for matrices and selfadjoint or non-selfadjoint differential equations i. Russian Math. Surveys 15 (1960), 1-74.

[24] Walker, R. Algebraic curves. Dover, 1962.

[25] Wasow, W. Asymptotic Expansions for Ordinary Differential Equations. Robert E. Krieger Publishing, 1967.

[26] WiLKInSON, J. The algebraic eigenvalue problem. Oxford, 1965.

[27] Wolovich, W. Linear Multivariable Systems. Springer, Berlin-New York, 1974. 